\title{
Velocity-modulation control of electron-wave propagation in graphene
}

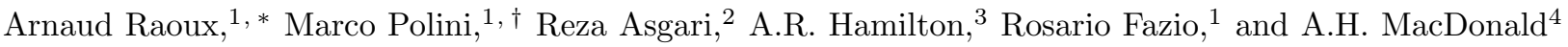 \\ ${ }^{1}$ NEST-CNR-INFM and Scuola Normale Superiore, I-56126 Pisa, Italy \\ ${ }^{2}$ School of Physics, Institute for Research in Fundamental Sciences (IPM), Tehran 19395-5531, Iran \\ ${ }^{3}$ School of Physics, University of New South Wales, Sydney NSW 2052, Australia \\ ${ }^{4}$ Department of Physics, University of Texas at Austin, Austin, Texas 78712, USA
}

\begin{abstract}
Wave propagation control by spatial modulation of velocity has a long history in optics and acoustics. We address velocity-modulation control of electron wave propagation in graphene and other two-dimensional Dirac-electron systems, pointing out a key distinction of the Dirac-wave case. We also propose a strategy for pattern transfer from a remote metallic layer that is based on manybody velocity renormalization.
\end{abstract}

PACS numbers: 73.23.Ad,71.10.-w,78.67.Pt

Introduction - Control of electromagnetic and mechanical wave propagation by spatial modulation of wave velocity [1, 2] has been studied for many decades in optics and acoustics, originally in relatively simple multilayer structures which have a wealth of practical applications and more recently in sophisticated two- and three-dimensional photonic and phononic crystal structures which can have gaps between transmission bands.

Recent advances [3] in the isolation and control of single and few-layer graphene electron systems motivate a close examination of velocity-modulation control in this material. Graphene is an allotrope of carbon atoms tightly packed in a two-dimensional (2D) honeycomb lattice. At energies near the Fermi energy of a neutral system, electron waves in graphene are described by a $2 \mathrm{D}$ massless Dirac equation and, like electromagnetic and mechanical waves, travel with a velocity that is independent of wavelength. Because of this property, the analogies of electron transport with both optics 4 and acoustics are stronger than in the conventional non-relativistic electron-wave case 5. In this article we first consider the propagation of massless Dirac fermion (MDF) waves through a medium with a position-dependent velocity, highlighting a key distinction between a Dirac wave and electromagnetic or acoustic waves. We then discuss a non-invasive strategy for achieving velocity modulation in graphene without any direct physical contact to the sample, by transferring a spatial pattern from remote metal layers via many-body velocity renormalization.

Scattering of MDFs against a velocity barrier - The influence of velocity variation on propagation is best illustrated by the simplest example, transmission through a velocity barrier 6, as illustrated in the inset in Fig. 1. We first solve this scattering problem, highlighting a key distinction between a Dirac wave and electromagnetic or acoustic waves. We consider MDFs in a medium in which the Fermi velocity $v$ changes as a function of the 2D position $\boldsymbol{r}: v=v(\boldsymbol{r})$. The massless Dirac equation in this case reads [7]

$$
\hat{\mathcal{H}} \Psi(\boldsymbol{r})=-i \hbar \sqrt{v(\boldsymbol{r})} \boldsymbol{\sigma} \cdot \nabla_{\boldsymbol{r}}[\sqrt{v(\boldsymbol{r})} \Psi(\boldsymbol{r})]=E \Psi(\boldsymbol{r}),
$$

where $\Psi(\boldsymbol{r})=\left(\Psi_{\mathrm{A}}(\boldsymbol{r}), \Psi_{\mathrm{B}}(\boldsymbol{r})\right)^{\mathrm{T}}$ is a two-component spinor, $\Psi_{\mathrm{A}}(\boldsymbol{r})$ and $\Psi_{\mathrm{B}}(\boldsymbol{r})$ are the honeycomb sublattice components of the electron wave, and $\boldsymbol{\sigma}=\left(\sigma_{x}, \sigma_{y}\right)$ is a 2D Pauli matrix vector. In using the Dirac equation we are assuming that velocity variations are slow on a lattice constant scale. In this limit spin and valley degrees of freedom play a passive role. Note that the Hamiltonian in Eq. (1) does not differ from its uniform system counterpart $\mathcal{H}_{\mathrm{MDF}}=-i \hbar v \boldsymbol{\sigma} \cdot \nabla_{\boldsymbol{r}}$ merely by the replacement $v \rightarrow v(\boldsymbol{r})$ : as pointed out by Peres in Ref. 7 this prescription would lead to a non-Hermitian operator. It is nevertheless convenient to introduce the auxiliary spinor $\Phi(\boldsymbol{r})=\sqrt{v(\boldsymbol{r})} \Psi(\boldsymbol{r})$ which satisfies

$$
-i \hbar v(\boldsymbol{r}) \boldsymbol{\sigma} \cdot \nabla_{\boldsymbol{r}} \Phi(\boldsymbol{r})=E \Phi(\boldsymbol{r}) .
$$

For the barrier problem illustrated in Fig. 1 $v(\boldsymbol{r})=v(x)$ changes only along the $\hat{\boldsymbol{x}}$ direction and momentum along the $\hat{\boldsymbol{y}}$ direction is a good quantum number.

We solve Eq. (2) for a simple velocity barrier

$$
v(x)=\left\{\begin{array}{l}
v_{1}, \text { if } x<0 \\
v_{2}, \text { if } 0<x<D . \\
v_{1}, \text { if } x>D
\end{array} .\right.
$$

This step-like model is justified when the Fermi wavelength $\lambda$ is much larger than the characteristic width over which $v(x)$ changes, as discussed at length for the potential-barrier case in Ref. 8 . For a given $\hat{\boldsymbol{y}}$ direction wavevector $k_{y}$ we are left with two coupled onedimensional first-order differential equations:

$$
-i \hbar v(x)\left(\frac{d}{d x} \mp k_{y}\right) \phi_{\mathrm{A}(\mathrm{B})}(x)=E \phi_{\mathrm{B}(\mathrm{A})}(x) .
$$

where $\Phi(\boldsymbol{r})=\phi(x) e^{i k_{y} y}$ and the spinor $\phi(x)=$ $\left(\phi_{\mathrm{A}}(x), \phi_{\mathrm{B}}(x)\right)^{\mathrm{T}}$. The first order equations for the spinor components can [because $v(x)$ is piecewise constant] be combined into a second order equation satisfied by both:

$$
\left\{\frac{d^{2}}{d x^{2}}+\left[\frac{E}{\hbar v(x)}\right]^{2}-k_{y}^{2}\right\} \phi_{i}(x)=0 .
$$




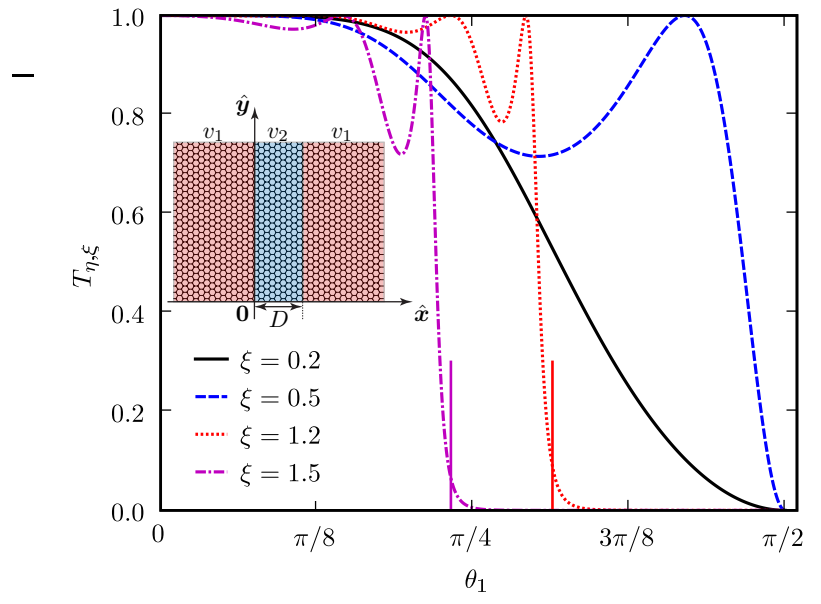

FIG. 1: (Color online) Inset: Cartoon of a velocity barrier. The velocity of the massless carriers changes along the $\hat{\boldsymbol{x}} \mathrm{di}-$ rection according to the simple functional form defined by Eq. (3). Main panel: Transmission probability $T_{\eta, \xi}\left(\theta_{1}\right)$ as a function of angle of incidence $\theta_{1}$ for velocity ratio $\xi=v_{2} / v_{1}$ equal to $0.2,0.5,1.2$, and 1.5 . The angle of incidence is defined so that $\theta_{1}=0$ corresponds to propagation perpendicular to the interface. These data have been obtained for $k D=\eta=4 \pi$ where $k$ is the incoming wavevector and $D$ is the barrier thickness. This value is typical 8 ] and corresponds for example to $k=2 \pi / \lambda$ with $\lambda=50 \mathrm{~nm}$ and $D=100 \mathrm{~nm}$. Note that for $\xi>1$, the transmission probability drops rapidly toward zero when the critical incidence angles, indicated by vertical bars, are exceeded. $\left(\theta_{\mathrm{c}} \approx 0.99 \mathrm{rad}\right.$ for $\xi=1.2$ and $\approx 0.73 \mathrm{rad}$ for $\xi=1.5$.)

The solutions of Eq. (5) can be written as

$$
\phi_{\mathrm{A}}(x)= \begin{cases}\left(e^{i k_{x} x}+r e^{-i k_{x} x}\right), & \text { if } x<0 \\ \left(a e^{i q_{x} x}+b e^{-i q_{x} x}\right), & \text { if } 0<x<D \\ t e^{i k_{x} x}, & \text { if } x>D\end{cases}
$$

where the constants $a, b, r$, and $t$ are to be determined,

$$
\left\{\begin{array}{l}
k_{x}=\sqrt{\left(\frac{E}{\hbar v_{1}}\right)^{2}-k_{y}^{2}}=k \cos \left(\theta_{1}\right) \\
q_{x}=\sqrt{\left(\frac{E}{\hbar v_{2}}\right)^{2}-k_{y}^{2}}=k \sqrt{\frac{1}{\xi^{2}}-\sin ^{2}\left(\theta_{1}\right)}
\end{array},\right.
$$

$k=E /\left(\hbar v_{1}\right), \xi=v_{2} / v_{1}$ is the velocity ratio, and $\theta_{1}$ is the angle of incidence, i.e. $\tan \left(\theta_{1}\right)=k_{y} / k_{x}$. The corresponding expression for $\phi_{\mathrm{B}}(x)$ can be obtained from Eq. (6) using Eq. (4). In Eq. (7) we can identify $\theta_{2}=\tan ^{-1}\left(k_{y} / q_{x}\right)$ as the angle of refraction and thereby obtain a quantum version of the famous Snell-Descartes law of geometrical optics:

$$
\frac{\sin \left(\theta_{1}\right)}{\sin \left(\theta_{2}\right)}=\frac{v_{1}}{v_{2}}=\frac{1}{\xi} .
$$

For $\xi>1$ and $\theta_{1}>\theta_{c}=\sin ^{-1}(1 / \xi)$, the classical total internal reflection angle, $q_{x}$ is imaginary and we expect negligible transmission through thick barriers. In this case the classical correspondence fails and the refraction angle is not well defined.

Explicit evaluation of the four coefficients in Eq. (6) requires matching conditions at the two interfaces which we obtain by the following argument. Dividing both sides of Eq. (2) by $v(x)$ and integrating across either interface implies that the auxiliary spinor $\Phi$ is continuous, and therefore that the physical $\Psi$ satisfies the following matching conditions:

$$
\left\{\begin{array}{l}
\Psi\left(0^{+}, y\right)=\frac{1}{\sqrt{\xi}} \Psi\left(0^{-}, y\right) \\
\Psi\left(D^{+}, y\right)=\sqrt{\xi} \Psi\left(D^{-}, y\right)
\end{array}\right.
$$

These discontinuities in $\Psi$ guarantee that the divergence of the local current $\boldsymbol{J}(\boldsymbol{r})=v(\boldsymbol{r}) \Psi^{\dagger}(\boldsymbol{r}) \boldsymbol{\sigma} \Psi(\boldsymbol{r})$ vanishes. Using these matching conditions we are able to obtain an explicit expression for the transmission 9 probability $T_{\eta, \xi}\left(\theta_{1}\right)=|t|^{2}:$

$$
T_{\eta, \xi}\left(\theta_{1}\right)=\frac{\cos ^{2}\left(\theta_{1}\right)\left[1-\xi^{2} \sin ^{2}\left(\theta_{1}\right)\right]}{C_{\eta, \xi}\left(\theta_{1}\right)},
$$

where $\eta=k D$ and $C_{\eta, \xi}\left(\theta_{1}\right)=\cos ^{2}\left(\theta_{1}\right)\left[1-\xi^{2} \sin ^{2}\left(\theta_{1}\right)\right]+$ $(1-\xi)^{2} \sin ^{2}\left(q_{x} \eta / k\right) \sin ^{2}\left(\theta_{1}\right)$.

In Fig. 1 we plot the transmission probability $T_{\eta, \xi}$ as a function of $\theta_{1}$ for $\eta=4 \pi$ at several different values of the velocity ratio $\xi$. Note that a velocity barrier is always perfectly transparent, $T_{\eta, \xi} \equiv 1$, for normal incidence $\left(\theta_{1}=0\right)$ as in the standard Klein problem [8]. This property of Dirac-wave propagation through a velocity barrier establishes a qualitative difference between the present case and the familiar electromagnetic and acoustic cases, and opens up new ground for the invention of spatial patterns with desirable transmission properties. It is also an important addition to the obvious difference in velocity-wavelength relationship in distinguishing Dirac-wave propagation from Schrödinger-wave propagation. In Fig. 2 we plot the integrated transmission,

$$
\mathcal{T}_{\eta}(\xi)=\frac{2}{\pi} \int_{0}^{\pi / 2} d \theta_{1} T_{\eta, \xi}\left(\theta_{1}\right)
$$

as a function of the velocity ratio $\xi$. A sharp change in behavior is visible at $\xi=1$ which foreshadows the total internal reflection properties of classical waves. Indeed, in the $\eta \rightarrow \infty$ limit it is easy to prove that for $\xi=1^{+}$ $\mathcal{T}_{\eta}(\xi) \rightarrow 2 \theta_{\mathrm{c}} / \pi \rightarrow 1-2 \sqrt{2} \sqrt{\xi-1} / \pi$, which is a nonanalytic function of $\xi$. For this reason even a slight mismatch in velocities can produce a large electron transport signal.

Molding electron flow- Doped or gated graphene sheets are normal Fermi liquids [10, 11] but have a number of unusual quantitative features [12] in their correlation physics which might provide an attractive route to velocity modulation, as we now explain. We first consider a grounded metal plane placed close to a graphene 


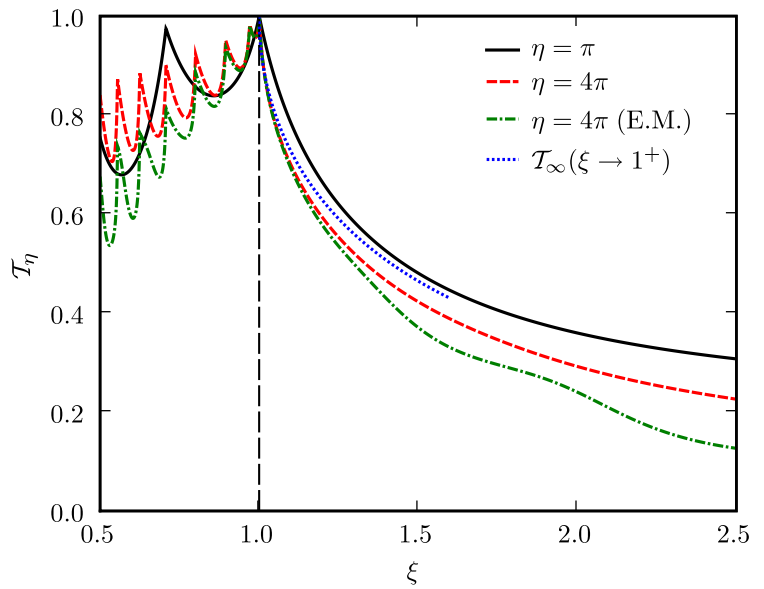

FIG. 2: (Color online) The integrated transmission $\mathcal{T}_{\eta}(\xi)$ as a function of the velocity ratio $\xi$ for $\eta=\pi$ [solid (black) line] and $\eta=4 \pi$ [dashed (red) line]. The dotted (blue) line shows the classical limit $\mathcal{T}_{\infty}\left(\xi \rightarrow 1^{+}\right)$. The dashdotted (green) line shows the integrated transmission for a linearly-polarized (along the $\hat{z}$-direction) electromagnetic wave scattering against a non-magnetic barrier (in this case $\xi=\sqrt{\epsilon_{1} / \epsilon_{2}}$, where $\epsilon_{i}$ are the dielectric constants of the media composing the barrier, and $\eta=\omega D / c)$.

sheet. The presence of the metal does not directly shift the chemical potential, like a biased gate would, but because electron-electron interactions between MDFs are expected to be substantially screened and thus weaker, has a rather large impact [13] on the renormalized Fermi velocity of the quasiparticles close to the Fermi energy that are important in transport. We show below that quasiparticles under the screening plane move at a speed $v^{\star}$ that is smaller than in an isolated graphene sheet [14, 15]. Using a single ground metal plane located close to a graphene sheet will thus lead to a very simple realization of the velocity barrier illustrated in the inset in Fig. 1 with $\xi<1$. To realize a velocity barrier with $\xi>1$ one instead needs to use two metal gates located on top of the regions with $x<0$ and $x>D$. The area on top of the strip $0<x<D$ must instead be left empty. In order for our abrupt-interface velocity-barrier calculation to be relevant, the distance to the metal gate $d$ would have to be smaller than the Fermi wavelength $\lambda$, but transmission properties will be similar even if this condition is not satisfied. Any shape of velocity modulation can be achieved by transferring a suitable spatial pattern from the remote lithographically-designed metal layer.

The effect of a metal gate on the quasiparticle velocity $v^{\star}$ can be estimated quantitatively by evaluating the quasiparticle self-energy $\Sigma$ of an interacting MDF system near the quasiparticle pole. We have generalized the GW theory [16] calculations described in Ref. 14, replacing the bare Coulomb interaction with the corresponding expression appropriate for a $2 \mathrm{D}$ electron system close to a perfect metallic screening plane: $V_{d}(q)=$ $2 \pi e^{2}[1-\exp (-2 q d)] / q[17$.

In Fig. 3 a) we report numerical results for $v^{\star} / v$ as a function of electron density $n$ for several values of $d$, and in Fig. 3p) as a function of $d$ for fixed density. We see that a substantial velocity contrast can be induced by metallic gates that are tens of nm's from the graphene plane. The effect of the gate can extend much further if it is separated from the graphene by a dielectric with $\epsilon_{\mathrm{r}} \gg 1$.

In addition to the velocity modulation the remote metallic layer will produce a shift in chemical potential. The reason lies in the fact that the Fermi energy quasiparticles whose transmission properties we study here satisfy, strictly speaking, a Dyson equation [14, not a single-particle massless Dirac equation. The chemical potential shift acts on the quasiparticles exactly like an external potential acts on a free particle. These shifts are small however, and can be compensated by biasing the patterned metallic plane although it might be difficult to completely separate velocity patterning from potential patterning in real experiments.

Discussion - Most of the considerations outlined in this paper apply equally well to any system in which matter waves satisfy a massless Dirac equation, for example to the surface states of topological insulators [18. Recently it has been proposed [19, 20, 21 that MDFs can also be realized in any standard 2D electron gas (2DEG), when appropriately nanopatterned. Similar proposals to realize the Dirac spectrum have been discussed in the contexts of ultracold atoms in optical lattices 22 and photonic crystals [23. Velocity modulation can be realized in these systems as well: a miniband structure is imprinted on a 2DEG subjected to a long-wavelength periodic external potential (i.e. a lateral superlattice) with hexagonal symmetry. If suitable conditions are satisfied [20], isolated Dirac points described by simple MDF Hamiltonians can appear in this miniband structure. As shown in Ref. 20 the Fermi velocity in these systems is quite sensitive to the strength of the periodic potential. By patterning the surface of a 2DEG in such a way to create three regions along a given direction in which the strength of the external periodic potential changes one can achieve a velocity barrier similar to the one sketched in the inset in Fig. 1.

In summary, we have calculated the transmission probability of massless Dirac fermions through a model "velocity barrier" and showed how electrons flowing through it obey the Snell-Descartes law of optics. We have also discussed a practical strategy for achieving substantial velocity modulation without damaging the graphene by exploiting the influence of a remote metallic layer on many-body renormalization of the quasiparticle velocity. Acknowledgements - A.R. and M.P. acknowledge useful 

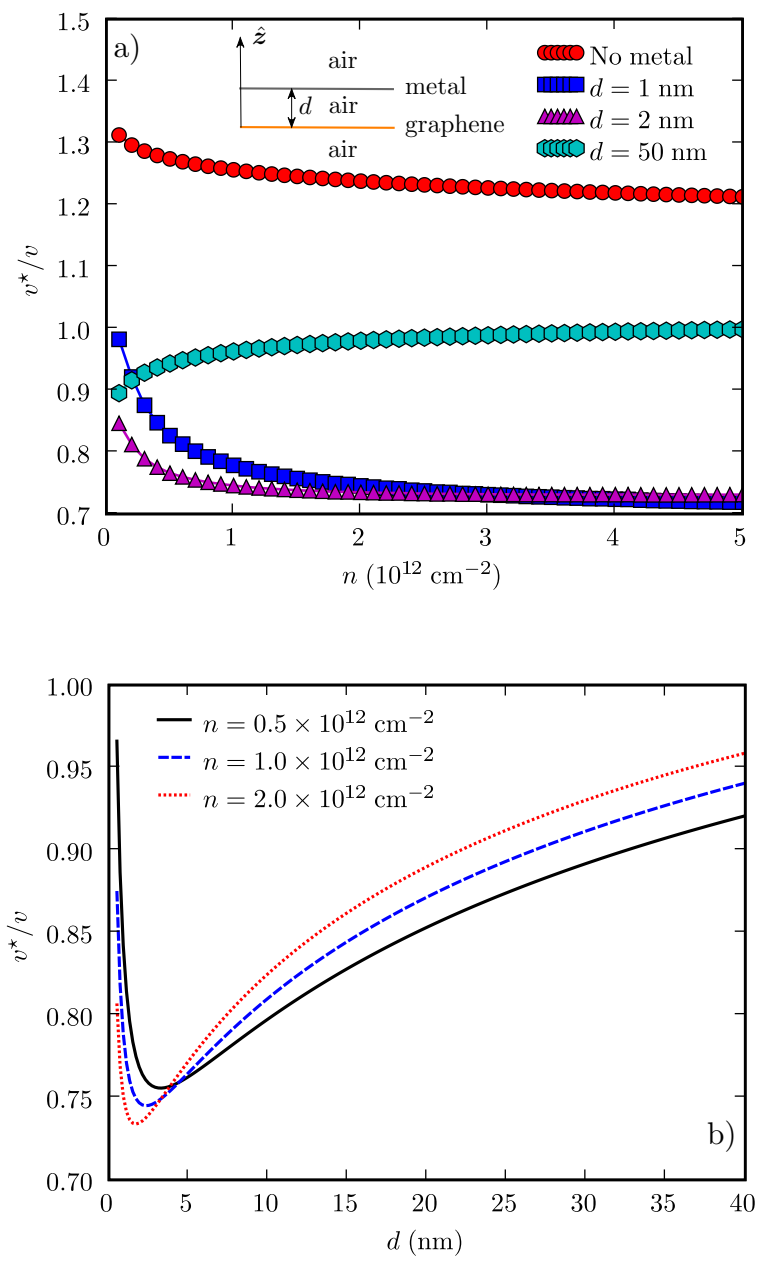

FIG. 3: (Color online) Panel a) Renormalized quasiparticle velocity $v^{\star}$ (in units of the bare velocity $v$ ) in graphene as a function of the electron density $n$ (in units of $10^{12} \mathrm{~cm}^{-2}$ ) in the presence of a ground perfect-metal plane located at a distance $d$ from the sheet (see inset). The (red) filled circles refer to an isolated sheet (in the absence of the perfect-metal plane). The other data refer to finite and increasingly larger (from top to bottom) values of $d$. Note how $v^{\star} / v$ is largely affected by the presence of the ground metal plane. Panel b) shows $v^{\star} / v$ as a function of $d$ (in $\mathrm{nm}$ ) for different values of $n: v^{\star} / v$ is largely suppressed even when the gate is quite far away from the graphene sheet.

conversations with Diego Rainis. M.P. and A.R.H. acknowledge support from the Gordon Godfrey bequest and the ARC APF scheme respectively. A.H.M. acknowledges support from SWAN and the NSF-NRI program.

* Permanent address: Formation Interuniversitaire de Physique, Département de Physique de l'École Normale Supérieure, 24 rue Lhomond, 75231 Paris Cedex 05,
France

$\dagger$ Electronic address: m.polini@sns.it

[1] J.D. Joannopoulos, R.D. Meade, and J.N. Winn, Photonic crystal: molding the flow of light, (Princeton University Press, Princeton NJ, 1995) and work cited therein.

[2] See for example M.M Sigalas and E.N Economou, Solid State Commun. 86, 141 (1993).

[3] A.H. Castro Neto et al., Rev. Mod. Phys. 81, 109 (2009).

[4] See for example V.V. Cheianov, V.I. Fal'ko, and B.L. Altshuler, Science 315, 1252 (2007); C.W. Beenakker et al., Phys. Rev. Lett. 102, 146804 (2009).

[5] For a review see B.J. LeRoy, J. Phys.: Condens. Matter 15, R1835 (2003).

[6] While this work was being written we learned that scattering of MDFs against a velocity barrier has also been studied by A. Concha and Z. Tešanovic, arXiv:0912.0493 1 .

[7] N.M.R. Peres, J. Phys: Condens. Matter 21, 095501 (2009); a position-dependent velocity has also been considered by F. de Juan, A. Cortijo, and M.A.H. Vozmediano, Phys. Rev. B 76, 165409 (2007) in the context of curved graphene.

[8] M.I. Katsnelson, K.S. Novoselov, and A.K. Geim, Nature Phys. 2, 620 (2006); for recent experimental studies of Klein tunneling see A.F. Young and P. Kim, Nature Phys. 5, 222 (2009); N. Stander, B. Huard, and D. GoldhaberGordon, Phys. Rev. Lett. 102, 026807 (2009).

[9] The reflection coefficient $r$ in Eq. (6) is given by $r_{\eta, \xi}\left(\theta_{1}\right)=(\xi-1) \exp \left(i \theta_{1}\right) \sin \left(q_{x} \eta / k\right) \sin \left(\theta_{1}\right) / D_{\eta, \xi}\left(\theta_{1}\right)$, with $D_{\eta, \xi}\left(\theta_{1}\right)=\left(\xi q_{x} / k\right) \cos \left(q_{x} \eta / k\right) \cos \left(\theta_{1}\right)-$ $i \sin \left(q_{x} \eta / k\right)\left[1-\xi \sin ^{2}\left(\theta_{1}\right)\right]$.

[10] M. Polini et al., Phys. Rev. B 77, 081411(R) (2008).

[11] E.H. Hwang and S. Das Sarma, Phys. Rev. B 77, 081412(R) (2008).

[12] Y. Barlas et al., Phys. Rev. Lett. 98, 236601 (2007).

[13] The impact of screening due to metal gates or highdensity 2D electron gases on Fermi-liquid parameters has been recently studied experimentally in the context of the metal-insulator transition: L.H. Ho et al., Phys. Rev. B 77, 201402(R) (2008).

[14] M. Polini et al., Solid State Commun. 143, 58 (2007).

[15] G. Li, A. Luican, and E.Y. Andrei, Phys. Rev. Lett. 102, 176804 (2009).

[16] See e.g. G.F. Giuliani and G. Vignale, Quantum Theory of the Electron Liquid (Cambridge University Press, Cambridge, 2005), Chapter 8.

[17] Note that $V_{d}(q)$ is simply the Fourier transform of $V_{d}(\rho, z)=-e \Phi(\rho, z)=e^{2}\left(\rho^{2}+z^{2}\right)^{-1 / 2}-e^{2}\left[\rho^{2}+(z-\right.$ $\left.2 d)^{2}\right]^{-1 / 2}$ evaluated at $z=0$ (where the graphene sheet is located). The electrostatic potential $\Phi(\rho, z)$ can be simply obtained from the method of image charges and satisfies the boundary condition $\Phi(\rho, d)=0$. This simple form of $V_{d}(q)$ is true if no dielectric media other than air are located underneath or above the graphene flake [see inset in Fig. [3 )]. For the sake of simplicity the data reported in Fig. 3 have been obtained for such suspended sheets [K.I. Bolotin et al., Solid State Commun. 146, 351 (2008); X. Du et al., Nature Nanotech. 3, 491 (2008)]. In this case, apart from electron density $n$ and metal-graphene distance $d$, the only parameter that controls $v^{\star} / v$ is the fine structure constant [3] $\alpha_{\mathrm{ee}}=e^{2} /(\hbar v) \simeq 2.2$.

[18] J. Moore, Nature Phys. 5, 378 (2009); Y. Xia et al., Na- 
ture Phys. 5, 398 (2009); H. Zhang et al., ibid. 5, 438 (2009).

[19] C.-H. Park and S.G. Louie, Nano Lett. 9, 1793 (2009).

[20] M. Gibertini et al., Phys. Rev. B 79, 241406(R) (2009).

[21] See also C. Flindt, N.A. Mortensen, and A.-P. Jauho, Nano Lett. 5, 2515 (2005) for a discussion of antidot lateral superlattices for quantum computation.
[22] S.L. Zhu, B. Wang, and L.M. Duan, Phys. Rev. Lett. 98, 260402 (2007); B. Wunsch, F. Guinea, and F. Sols, New J. Phys. 10, 103027 (2008).

[23] F.D.M. Haldane and S. Raghu, Phys. Rev. Lett. 100, 013904 (2008); R.A. Sepkhanov, J. Nilsson, and C.W.J. Beenakker, Phys. Rev. B 78, 045122 (2008). 